Supporting Information: Org.Lett, One-pot synthesis of homoallylic ketones. Hansford et al.

\title{
One-Pot Synthesis of Homoallylic Ketones from the Addition of Vinyl Grignard Reagent to Carboxylic Esters
}

\author{
Karl A. Hansford, James E. Dettwiler and William D. Lubell ${ }^{*}$ \\ Département de Chimie, Université de Montréal, C.P. 6128, Succursale Centre-Ville, Montréal, Québec, \\ Canada. H3C $3 J 7$ \\ lubell@chimie.umontreal.ca
}


Supporting Information: Org.Lett, One-pot synthesis of homoallylic ketones. Hansford et al.

\section{General Experimental Methods.}

For reactions performed under anhydrous conditions, glassware was either oven- or flame-dried and the reaction was run under a positive pressure of argon. Tetrahydrofuran was freshly distilled from sodium/benzophenone ketyl. Vinylmagnesium bromide reagent was either used as supplied (Aldrich) as a $1 M$ solution in THF, or freshly prepared ${ }^{1}$ as a solution in THF from Mg (Aldrich; $99.98 \%$ purity) and vinyl bromide (Aldrich; $98 \%$ ). The Grignard reagents were titrated against menthol and 2,2'-dipyridyl. CuCN was used as supplied (Aldrich; $99 \%$ purity) and $\mathrm{Cu}(\mathrm{OAc})_{2} \cdot \mathrm{H}_{2} \mathrm{O}$ (J.T. Baker; $99.7 \%$ purity) was recrystallized from hot aqueous acetic acid solution $(50 \% \mathrm{v} / \mathrm{v})$ prior to use. Anhydrous $\mathrm{Cu}(\mathrm{OAc})_{2}$ was prepared by heating freshly recrystallized $\mathrm{Cu}(\mathrm{OAc})_{2} \cdot \mathrm{H}_{2} \mathrm{O}$ in-vacuo (150 $\left.{ }^{\circ} \mathrm{C}\right)$ until a constant weight was achieved. Amino acids were obtained from Aldrich (threonine and alanine) or Avocado Research Chemicals (proline and serine), and were $N$-protected using standard procedures. (2RS)- and (2S)-1-benzenesulfonyl-pyrrolidine-2carbonyl chloride was synthesized as previously described. ${ }^{2}$ Flash chromatography was performed using silica gel (Silicycle; 230-400 mesh), neutral alumina (Aldrich; Brockmann I, 150 mesh), basic alumina (Acros; 50-200 mesh) and Florisil (Aldrich; 100200 mesh). Analytical thin-layer chromatography was conducted with Silicycle silica gel $\mathrm{F}_{254}$ plates. NMR spectra were run at $400 \mathrm{MHz}$ on a Bruker AV400. Chemical shifts $\left(\delta_{\mathrm{H}}\right)$ are reported in parts per million (ppm) and are referenced to the residual solvent peak. HRMS (EI and FAB) were obtained by the Université de Montréal Mass Spectroscopy facility. The reported yields are the actual isolated yields of purified material and are not optimized. 
Supporting Information: Org.Lett, One-pot synthesis of homoallylic ketones. Hansford et al.

\section{Procedure A: Preparation of compounds (2a, 2b, 2e, 2k, 2o)}

Vinylmagnesium bromide reagent was added via syringe to a flame-dried two-neck flask. With rapid stirring, the reagent was cooled to $-45^{\circ} \mathrm{C}$ (dry ice/acetonitrile). On occasion, a little THF was added to the flask to facilitate stirring. While maintaining a positive pressure of argon, solid $\mathrm{Cu}(\mathrm{OAc})_{2}$ was added as quickly as possible to the reaction flask, and the flask was re-sealed. After stirring for $10 \mathrm{~min}$, a solution of the ester in THF (2 mL per $1 \mathrm{mmol}$ ester) was added over $5 \mathrm{~min}$, and the resultant mixture was stirred for $1 \mathrm{~h}$. The cold bath was then removed and replaced with an ice bath, and stirring maintained at $0{ }^{\circ} \mathrm{C}$ for $1 \mathrm{~h}$. With the ice bath in place, the reaction mixture was allowed to slowly warm to room temperature with overnight stirring. The reaction mixture was quenched by treatment with saturated $\mathrm{NH}_{4} \mathrm{Cl}$ solution $(200 \% \mathrm{v} / \mathrm{v}$ based on total reaction volume) at 0 ${ }^{\circ} \mathrm{C}$. After vigorous shaking, the layers were separated, and the aqueous phase extracted with $\mathrm{Et}_{2} \mathrm{O}$ or EtOAc. The combined extracts were washed with saturated $\mathrm{NH}_{4} \mathrm{Cl}$ solution (x 1), saturated $\mathrm{NaHCO}_{3}$ solution (x 1), pH 6.8 phosphate buffer (x 1), brine (x 1), dried $\left(\mathrm{MgSO}_{4}\right)$ and evaporated. The crude product was then purified as specified.

Note: The $\mathrm{Cu}(\mathrm{OAc})_{2}$ could also be pre-dissolved with the ester in THF prior to reaction with the Grignard reagent at $-45^{\circ} \mathrm{C}$ with similar outcome.

\section{Procedure B: Preparation of compound (2d)}

Identical to general procedure A, with the exception that after stirring the reaction mixture at $-45^{\circ} \mathrm{C}$ for $1 \mathrm{~h}$, stirring was maintained at $0{ }^{\circ} \mathrm{C}$ for the specified time, and then worked up as in procedure A. The crude product was purified as specified. 
Supporting Information: Org.Lett, One-pot synthesis of homoallylic ketones. Hansford et al.

\section{Procedure C: Preparation of compounds (2f, 2g)}

Identical to procedure A, with the following exceptions- 1: after completion of the reaction, $\mathrm{NaH}_{2} \mathrm{PO}_{4}$ solution (200\% v/v based on total reaction volume; $2 M$ aqueous) was substituted for saturated $\mathrm{NH}_{4} \mathrm{Cl}$ solution as quenching reagent; 2: in the case of compound 2f, stirring was maintained at $0{ }^{\circ} \mathrm{C}$ for $15 \mathrm{~min}$ before workup. The crude product was then purified as specified.

\section{Procedure D: Preparation of compounds (2i, 2l, 2n)}

Identical to procedure A, with the exception that the reaction was run in the absence of copper catalyst. The crude product was then purified as specified.

\section{Procedure E: Preparation of compound (2m)}

Identical to procedure A, with the following exceptions- 1: the reaction was run in the absence of copper catalyst and, 2: after completion of the reaction, $\mathrm{pH} 6.8$ phosphate buffer solution ( $200 \% \mathrm{v} / \mathrm{v}$ based on total reaction volume) was substituted for saturated $\mathrm{NH}_{4} \mathrm{Cl}$ solution as quenching reagent. The crude product was then purified as specified.

\section{Procedure F: Preparation of compounds (2c, 2j)}

A suspension of $\mathrm{CuCN}$ in $\mathrm{THF}(2 \mathrm{~mL}$ per $1 \mathrm{mmol} \mathrm{CuCN})$ was cooled to $-78{ }^{\circ} \mathrm{C}$, and vinylmagnesium bromide reagent was added over $10 \mathrm{~min}$, followed by a solution of the ester in THF ( $2 \mathrm{~mL}$ per $1 \mathrm{mmol}$ ester). With the cold bath in place, the resultant mixture 
Supporting Information: Org.Lett, One-pot synthesis of homoallylic ketones. Hansford et al.

was allowed to slowly warm to room temperature with overnight stirring. The reaction mixture was quenched by treatment with $\mathrm{HCl}$ solution (1M, aqueous) (200\% v/v based on total reaction volume) at $0{ }^{\circ} \mathrm{C}$. After vigorous shaking, the layers were separated, and the aqueous phase extracted with $\mathrm{Et}_{2} \mathrm{O}$ or EtOAc. The combined extracts were washed with saturated $\mathrm{NaHCO}_{3}$ solution (x 1), pH 6.8 phosphate buffer (x 1), brine (x 1), dried $\left(\mathrm{MgSO}_{4}\right)$ and evaporated. The crude product was then purified as specified.

\section{Procedure G: Preparation of compound (2h)}

Identical to procedure $\mathrm{F}$, with the following exception: after completion of the reaction, work up was achieved by pouring the crude reaction mixture onto a rapidly stirred mixture of $\mathrm{NaH}_{2} \mathrm{PO}_{4}$ solution $(2 \mathrm{M}$, aqueous; $100 \mathrm{~mL})$ and $\mathrm{Et}_{2} \mathrm{O}(100 \mathrm{~mL})$ at $0{ }^{\circ} \mathrm{C}$. After separation of the layers, the aqueous phase was extracted with $\mathrm{Et}_{2} \mathrm{O}$ ( $\mathrm{x} 2$ ), and the combined organic phase was washed with saturated $\mathrm{NaHCO}_{3}$ solution (x 1), brine (x 1), dried $\left(\mathrm{MgSO}_{4}\right)$ and evaporated. The crude product was then purified as specified.

\section{1-(1-Benzenesulfonyl-piperidin-4-yl)-pent-4-en-1-one (2a)}

Prepared from methyl ester 1a $(281 \mathrm{mg}, 1 \mathrm{mmol})$ and vinyl magnesium bromide $(4 \mathrm{mmol})$ in the presence of $\mathrm{Cu}(\mathrm{OAc})_{2}$

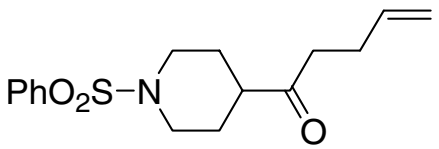
(0.5 mmol) according to procedure A (reaction time $12 \mathrm{~h}$ at room temperature) to yield crude product $(264 \mathrm{mg})$. Purification by chromatography over silica gel (40:60 $\mathrm{Et}_{2} \mathrm{O} /$ hexanes) gave $\mathbf{2 a}$ as a cream colored solid (135 mg, $44 \%$ ). Recrystallization from EtOAc/hexanes gave pearly leaflets, mp 82-84 ${ }^{\circ} \mathrm{C}$. TLC Rf 0.47 (20:80 EtOAc/hexanes). ${ }^{1} \mathrm{H}$ NMR (400 MHz, $\mathrm{CDCl}_{3}$ ) $\delta 1.67$ (dddd, J = 11.4, 11.4, 11.4, 4.0 Hz, 2H), 1.79-1.91 (m, 2H), 2.16-2.29 (m, 3H), 2.35 (dt, $J=11.7,2.3 \mathrm{~Hz}, 2 \mathrm{H}), 2.45$ (t, J = 7.4 Hz, 2H), 3.63$3.75(\mathrm{~m}, 2 \mathrm{H}), 4.86-5.0(\mathrm{~m}, 2 \mathrm{H}), 5.64-5.78(\mathrm{~m}, 1 \mathrm{H}), 7.43-7.61(\mathrm{~m}, 3 \mathrm{H}), 7.71(\mathrm{~d}, \mathrm{~J}=7.4$ 
Supporting Information: Org.Lett, One-pot synthesis of homoallylic ketones. Hansford et al.

$\mathrm{Hz}, 2 \mathrm{H}) .{ }^{13} \mathrm{C} \mathrm{NMR}\left(100 \mathrm{MHz}, \mathrm{CDCl}_{3}\right) \delta 210.5,136.8,135.9,132.8,129.0,127.5,115.3$, 47.2, 45.5, 39.5, 27.4, 26.8. HRMS (EI) $\mathrm{m} / \mathrm{z} 307.1238\left[\mathrm{M}^{+}\right.$; calcd for $\mathrm{C}_{16} \mathrm{H}_{21} \mathrm{NO}_{3} \mathrm{~S}$ : 307.1242].

\section{1-(4-Methoxy-phenyl)-hept-6-en-3-one (2b)}

Prepared from methyl ester $\mathbf{1 b}(408 \mathrm{mg}, 2.1 \mathrm{mmol})$ and vinyl magnesium bromide $(8.4 \mathrm{mmol})$ in the presence of $\mathrm{Cu}(\mathrm{OAc})_{2}$ $(0.63 \mathrm{mmol})$ according to procedure $\mathrm{A}$ (reaction time $12 \mathrm{~h}$ at

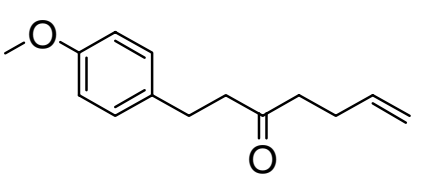
room temperature) to yield crude product $(475 \mathrm{mg})$. Purification by chromatography over basic alumina (activity grade I; 10:90 $\mathrm{Et}_{2} \mathrm{O} /$ hexanes, increasing to $40: 60 \mathrm{Et}_{2} \mathrm{O} /$ hexanes over $5 \%$ increments) gave $\mathbf{2 b}$ as a pale yellow oil (294 mg, $64 \%)$. TLC $\mathrm{R}_{\mathrm{f}} 0.33$ (10:90 $\mathrm{Et}_{2} \mathrm{O} /$ hexanes). ${ }^{1} \mathrm{H} \mathrm{NMR}\left(400 \mathrm{MHz}, \mathrm{CDCl}_{3}\right) \delta 2.25-2.34(\mathrm{~m}, 2 \mathrm{H}), 2.46(\mathrm{t}, J=7.4 \mathrm{~Hz}$, $2 \mathrm{H}), 2.68(\mathrm{t}, J=7.6 \mathrm{~Hz}, 2 \mathrm{H}), 2.82(\mathrm{t}, J=7.6 \mathrm{~Hz}, 2 \mathrm{H}), 3.75(\mathrm{~s}, 3 \mathrm{H}), 4.90-5.04(\mathrm{~m}, 2 \mathrm{H})$, 5.70-5.83 (m, 1H), $6.80(\mathrm{~d}, J=8.7 \mathrm{~Hz}, 2 \mathrm{H}), 7.08(\mathrm{~d}, J=8.7 \mathrm{~Hz}, 2 \mathrm{H}) .{ }^{13} \mathrm{C}$ NMR $(100$ $\left.\mathrm{MHz}, \mathrm{CDCl}_{3}\right) \delta 209.3,157.8,137.0,133.0,129.1,115.1,113.7,55.1,44.5,41.9,28.7$, 27.6. HRMS (EI) $\mathrm{m} / \mathrm{z} 218.1308\left[\mathrm{M}^{+}\right.$; calcd for $\mathrm{C}_{14} \mathrm{H}_{18} \mathrm{O}_{2}$ : 218.1307].

\section{1-Benzo[1,3]dioxol-5-yl-pent-4-en-1-one (2c)}

Prepared from methyl ester 1c (360 mg, $2 \mathrm{mmol})$ and vinyl magnesium bromide $(8.0 \mathrm{mmol})$ in the presence of $\mathrm{CuCN}(0.6$ $\mathrm{mmol}$ ) according to procedure $\mathrm{F}$ to yield crude product $(340 \mathrm{mg}$ ).

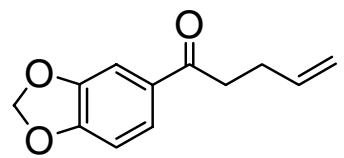
Purification by chromatography over silica gel (toluene) gave $\mathbf{2 c}$ as a pale yellow oil (208 $\mathrm{mg}, 51 \%$ ). TLC $\mathrm{R}_{\mathrm{f}} 0.37$ (10:90 Et $2 \mathrm{O} /$ hexanes). ${ }^{1} \mathrm{H}$ NMR (400 MHz, $\left.\mathrm{CDCl}_{3}\right) \delta$ 2.40-2.47 (m, 2H), $2.96(\mathrm{t}, J=7.4 \mathrm{~Hz}, 2 \mathrm{H}), 4.94-4.99(\mathrm{~m}, 1 \mathrm{H}), 5.04(\mathrm{dq}, J=16.0,1.7 \mathrm{~Hz}, 1 \mathrm{H})$, 5.79-5.92 (m, 1H), $6.0(\mathrm{~s}, 2 \mathrm{H}), 6.80(\mathrm{~d}, J=8.3 \mathrm{~Hz}, 1 \mathrm{H}), 7.40(\mathrm{~d}, J=2.0 \mathrm{~Hz}, 1 \mathrm{H}), 7.53$ $(\mathrm{dd}, J=8.3,2.0 \mathrm{~Hz}, 1 \mathrm{H}) .{ }^{13} \mathrm{C} \mathrm{NMR}\left(100 \mathrm{MHz}, \mathrm{CDCl}_{3}\right) \delta 197.4,151.6,148.1,137.3$, 131.7, 124.1, 115.2, 107.8, 101.8, 37.4, 28.3 (one line obscured). HRMS (EI) m/z 204.0792 [M+c calcd for $\left.\mathrm{C}_{12} \mathrm{H}_{12} \mathrm{O}_{3}: 204.0786\right]$. 
Supporting Information: Org.Lett, One-pot synthesis of homoallylic ketones. Hansford et al.

\section{1-Furan-2-yl-pent-4-en-1-one (2d)}

Prepared from methyl ester $\mathbf{1 d}(126 \mathrm{mg}, 1 \mathrm{mmol})$ and vinyl magnesium bromide $(4.0 \mathrm{mmol})$ in the presence of $\mathrm{Cu}(\mathrm{OAc})_{2}(0.5 \mathrm{mmol})$ according

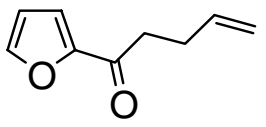
to procedure $\mathrm{B}$ (reaction time $1 \mathrm{~h}$ at $0{ }^{\circ} \mathrm{C}$ ) to yield crude product (122 mg). Purification by chromatography over neutral alumina (activity grade $\mathrm{I} ; 5: 95 \mathrm{Et}_{2} \mathrm{O} /$ hexanes) gave $\mathbf{2 d}$ as a yellow oil (72 mg, $48 \%$ ). $\mathrm{R}_{\mathrm{f}} 0.17$ (5:95 Et $\mathrm{E}_{2} \mathrm{O} / \mathrm{hexanes).}{ }^{1} \mathrm{H} \mathrm{NMR}\left(400 \mathrm{MHz}, \mathrm{CDCl}_{3}\right) \delta$ 2.38-2.50 (m, 2H), 2.89 (t, $J=7.8 \mathrm{~Hz}, 2 \mathrm{H}), 4.93-5.00(\mathrm{~m}, 1 \mathrm{H}), 5.00-5.10(\mathrm{~m}, 1 \mathrm{H}), 5.75-$ $5.92(\mathrm{~m}, 1 \mathrm{H}), 6.49(\mathrm{dd}, J=4.8,1.6 \mathrm{~Hz}, 1 \mathrm{H}), 7.15(\mathrm{dd}, J=4.8,0.9 \mathrm{~Hz}, 1 \mathrm{H}), 7.55$ (dd, $J=$ 1.6, $0.9 \mathrm{~Hz}, 1 \mathrm{H}) .{ }^{13} \mathrm{C} \mathrm{NMR}\left(100 \mathrm{MHz}, \mathrm{CDCl}_{3}\right) \delta 188.7,152.6,146.2,136.9,116.9,115.4$, 112.1, 37.5, 28.0. HRMS (EI) m/z 150.0681 [ $\mathrm{M}^{+}$; calcd for $\mathrm{C}_{9} \mathrm{H}_{10} \mathrm{O}_{2}$ : 150.0681].

\section{1-(1H-Indol-3-yl)-hex-5-en-2-one (2e)}

Prepared from methyl ester 1e (190 mg, $1 \mathrm{mmol})$ and vinyl magnesium bromide $(6 \mathrm{mmol})$ in the presence of $\mathrm{Cu}(\mathrm{OAc})_{2}(0.5$ mmol) according to procedure A (reaction time $12 \mathrm{~h}$ at room

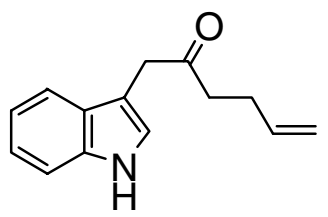
temperature) to yield crude product $(261 \mathrm{mg})$. Purification by chromatography over silica gel (15:85 EtOAc/hexanes) gave $\mathbf{2 e}$ as brown oil (79 mg, $37 \%)$. TLC $\mathrm{R}_{\mathrm{f}} 0.47$ (30:70 EtOAc/hexanes). ${ }^{1} \mathrm{H}$ NMR (400 MHz, $\left.\mathrm{CDCl}_{3}\right) \delta 2.23-2.34(\mathrm{~m}, 2 \mathrm{H}), 2.58(\mathrm{t}, J=7.4 \mathrm{~Hz}$, $2 \mathrm{H}), 3.81(\mathrm{~s}, 2 \mathrm{H}), 4.87-5.01(\mathrm{~m}, 2 \mathrm{H}), 5.67-5.81(\mathrm{~m}, 1 \mathrm{H}), 7.07-7.16(\mathrm{~m}, 2 \mathrm{H}), 7.20(\mathrm{t}, J=$ $8.0 \mathrm{~Hz}, 1 \mathrm{H}), 7.34$ (d, $J=8.0 \mathrm{~Hz}, 1 \mathrm{H}), 7.53$ (d, $J=7.7 \mathrm{~Hz}, 1 \mathrm{H}), 8.1-8.2$ (br s, $1 \mathrm{H}) .{ }^{13} \mathrm{C}$ NMR (100 MHz, $\left.\mathrm{CDCl}_{3}\right) \delta$ 208.6, 137.1, 136.1, 127.2, 123.1, 122.2, 119.8, 118.7, 115.1,

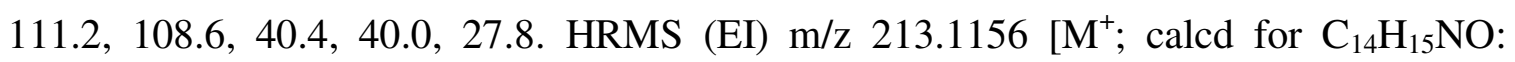
213.1154]. 
Supporting Information: Org.Lett, One-pot synthesis of homoallylic ketones. Hansford et al.

\section{1-Pyridin-3-yl-pent-4-en-1-one (2f)}

Prepared from methyl ester 1 f $(306 \mathrm{mg}, 2.2 \mathrm{mmol})$ and vinyl magnesium bromide $(6.6 \mathrm{mmol})$ according to procedure $\mathrm{C}$ (reaction time $4 \mathrm{~h}$ at $-45^{\circ} \mathrm{C}$ ) to yield crude product $(338 \mathrm{mg})$. Purification by

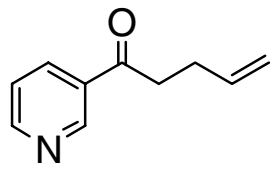
chromatography over basic alumina (activity grade I; 40:60 EtOAc/hexanes) gave $2 \mathbf{f}$ as a yellow oil (147 mg, $42 \%) . \mathrm{R}_{\mathrm{f}} 0.24$ (40:60 EtOAc/hexanes). ${ }^{1} \mathrm{H}$ NMR (400 MHz, $\mathrm{CDCl}_{3}$ ) $\delta$ 2.42-2.52 (m, 2H), $3.06(\mathrm{t}, J=7.4 \mathrm{~Hz}, 2 \mathrm{H}), 4.99$ (dq, $J=10.4,1.4 \mathrm{~Hz}, 1 \mathrm{H}), 5.04$ (dq, $J$ $=17.0,1.7 \mathrm{~Hz}, 1 \mathrm{H}), 5.79-5.92(\mathrm{~m}, 1 \mathrm{H}), 7.38(\mathrm{ddd}, J=8.0,2.0,1.9 \mathrm{~Hz}, 1 \mathrm{H}), 8.19(\mathrm{dt}, J=$ 8.0, $2.0 \mathrm{~Hz}, 1 \mathrm{H}), 8.71-8.77(\mathrm{~m}, 1 \mathrm{H}), 9.14(\mathrm{~d}, J=1.9 \mathrm{~Hz}, 1 \mathrm{H}) .{ }^{13} \mathrm{C}$ NMR $(100 \mathrm{MHz}$, $\left.\mathrm{CDCl}_{3}\right) \delta 198.1,153.4,149.6,136.7,135.3,132.0,123.6,115.6,38.0,27.7$. HRMS (EI) $\mathrm{m} / \mathrm{z} 161.0842$ [ $\mathrm{M}^{+}$; calcd for $\left.\mathrm{C}_{10} \mathrm{H}_{11} \mathrm{NO}: 161.0841\right]$.

\section{1-Phenyl-pent-4-en-1-one (2g)}

Prepared from methyl ester $\mathbf{1 g}(1.36 \mathrm{~g}, 10 \mathrm{mmol})$ and vinyl magnesium bromide $(30 \mathrm{mmol})$ according to procedure $\mathrm{C}$ to yield crude product $(1.65 \mathrm{~g})$. Short-path distillation gave the title compound as a pale

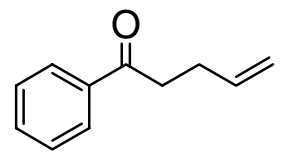
yellow oil that was contaminated with ca. $5 \%$ tertiary alcohol 3g (1.11 g, $69 \%)$. The contaminant could be removed by chromatography over basic alumina; thus a portion (210 mg) was chromatographed (10:90 $\mathrm{Et}_{2} \mathrm{O} /$ hexanes elution) to yield pure product (181 mg) as a colourless oil. TLC Rf 0.49 (10:90 Et2O/hexanes). 1H NMR (400 MHz, CDCl 3 ) $\delta$ 2.43-2.57 (m, 2H), $3.08(\mathrm{t}, J=7.4 \mathrm{~Hz}, 2 \mathrm{H}), 4.97-5.15(\mathrm{~m}, 2 \mathrm{H}), 5.84-5.98(\mathrm{~m}, 1 \mathrm{H}), 7.46$ $(\mathrm{t}, J=7.4 \mathrm{~Hz}, 2 \mathrm{H}), 7.56(\mathrm{t}, J=7.4 \mathrm{~Hz}, 1 \mathrm{H}), 7.97(\mathrm{~d}, J=7.4 \mathrm{~Hz}, 2 \mathrm{H}) .{ }^{13} \mathrm{C} \mathrm{NMR}(100$ $\left.\mathrm{MHz}_{\mathrm{CDCl}}\right) \delta 199.3,137.2,136.8,132.9,128.5,127.9,115.2,37.6,28.0$. HRMS (EI) $\mathrm{m} / \mathrm{z} 160.0895$ [M+c calcd for $\mathrm{C}_{11} \mathrm{H}_{12}$ : 160.0888$]$. 
Supporting Information: Org.Lett, One-pot synthesis of homoallylic ketones. Hansford et al.

tert-Butyl-(1R)-1-methyl-2-oxo-hex-5-enylcarbamate (2h)

Prepared from methyl ester $\mathbf{1 h}(1.0 \mathrm{mg}, 4.92 \mathrm{mmol})$ and vinyl magnesium bromide $(29.5 \mathrm{mmol})$ in the presence of $\mathrm{CuCN}(2.1$ mmol) according to procedure $\mathrm{G}$ to yield crude product (1.32 g).<smiles>C=CCCC(=O)C(C)N</smiles>
Purification by chromatography over basic alumina (activity grade III; 2.5:97.5 $\mathrm{Et}_{2} \mathrm{O} /$ toluene) gave $\mathbf{2 h}$ as a pale yellow oil (862 $\left.\mathrm{mg}, 77 \%\right)$. TLC $\mathrm{R}_{\mathrm{f}} 0.24$ (20:80 $\mathrm{Et}_{2} \mathrm{O} /$ hexanes). ${ }^{1} \mathrm{H} \mathrm{NMR}\left(400 \mathrm{MHz}, \mathrm{CDCl}_{3}\right) \delta 1.30$ (d, $\left.J=7.0 \mathrm{~Hz}, 3 \mathrm{H}\right), 1.41(\mathrm{~s}, 9 \mathrm{H})$, 2.26-2.38 (m, 2H), 2.48-2.68 (m, 2H), 4.22-4.34 (m, 1H), 4.92-5.07 (m, 2H), 5.24-5.34 $(\mathrm{m}, 1 \mathrm{H}), 5.70-5.84(\mathrm{~m}, 1 \mathrm{H}) .{ }^{13} \mathrm{C} \mathrm{NMR}\left(100 \mathrm{MHz}, \mathrm{CDCl}_{3}\right) \delta 208.8,155.1,136.7,115.4$, 79.6, 55.0, 38.1, 28.2, 27.4, 17.7. HRMS (EI) m/z 227.1523 [M+' calcd for $\mathrm{C}_{12} \mathrm{H}_{21} \mathrm{NO}_{3}$ : $227.1521] .[\alpha]_{\mathrm{D}}^{20}=-28^{\circ}\left(\mathrm{c} 0.01, \mathrm{CHCl}_{3}\right)$.

\section{(2S)-2-(9-Phenyl-9H-fluoren-9-ylamino)-hept-6-en-3-one (2i)}

Prepared from methyl ester 1i (343 mg, $1 \mathrm{mmol})$ and vinyl magnesium bromide $(6 \mathrm{mmol})$ according to procedure $\mathrm{D}$ (reaction time $48 \mathrm{~h}$ at room

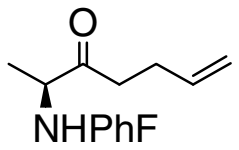
temperature) to yield crude product (324 mg). Purification by chromatography over silica gel gave (in order of elution) $\mathbf{2} \mathbf{i}$ as a pale yellow solid (96 mg, $26 \%$ ) followed by recovered starting material $(102 \mathrm{mg})$. Recrystallization of $\mathbf{2} \mathbf{i}$ from $\left({ }^{\mathrm{i}} \mathrm{Pr}\right)_{2} \mathrm{O}$ gave colorless blocks, mp 104-107 ${ }^{\circ} \mathrm{C}$. TLC $\mathrm{R}_{\mathrm{f}} 0.40$ (10:90 Et 2 O/hexanes). ${ }^{1} \mathrm{H}$ NMR (400 MHz, $\mathrm{CDCl}_{3}$ ) $\delta 0.97(\mathrm{~d}, J=7.1 \mathrm{~Hz}, 3 \mathrm{H}), 1.52-1.61(\mathrm{~m}, 1 \mathrm{H}), 1.81-2.03(\mathrm{~m}, 2 \mathrm{H}), 2.12-2.21(\mathrm{~m}, 1 \mathrm{H}), 2.68$ (q, $J=7.1 \mathrm{~Hz}, 1 \mathrm{H}), 3.44$ (br s, 1H), 4.81-4.84 (m, 1H), 4.86 (t, $J=1.3 \mathrm{~Hz}, 1 \mathrm{H}), 5.49-5.60$ $(\mathrm{m}, 1 \mathrm{H}), 7.05-7.42(\mathrm{~m}, 11 \mathrm{H}), 7.65-7.70(\mathrm{~m}, 2 \mathrm{H}) .{ }^{13} \mathrm{C} \mathrm{NMR}\left(100 \mathrm{MHz}, \mathrm{CDCl}_{3}\right) \delta 213.7$, $149.9,149.5,144.5,141.0,139.9,137.0,128.2$, 128.0, 127.7, 127.1, 126.4, 126.1, 125.3, 
Supporting Information: Org.Lett, One-pot synthesis of homoallylic ketones. Hansford et al.

$119.8,119.7,114.9,73.1,56.7,39.0,27.3,20.8$. HRMS (FAB) m/z $368.2022\left[\mathrm{M}+\mathrm{H}^{+}\right.$; calcd for $\left.\left[\mathrm{C}_{26} \mathrm{H}_{26} \mathrm{NO}\right]^{+}: 368.2009\right]$. $[\alpha]_{\mathrm{D}}^{20}=+242^{\circ}\left(\mathrm{c} 0.01, \mathrm{CHCl}_{3}\right)$.

tert-Butyl-(1S)-1-Hydroxymethyl-2-oxo-hex-5-enylcarbamate (2j)

Prepared from methyl ester $\mathbf{1 j}$ (877 $\mathrm{mg}, 4 \mathrm{mmol}$ ) and vinyl magnesium bromide $(24 \mathrm{mmol})$ in the presence of $\mathrm{CuCN}(1.2 \mathrm{mmol})$

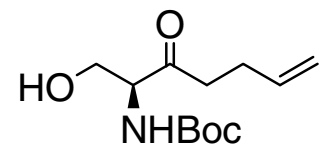
according to procedure $\mathrm{F}$ to yield crude product $(1.01 \mathrm{~g})$. Purification by chromatography over florisil (5:95 EtOAc/hexanes increasing to 100\% EtOAc in $5 \%$ increments) gave $\mathbf{2 j}$ as an oil (576 mg, 59\%). TLC $\mathrm{R}_{\mathrm{f}} 0.56$ (60:40 EtOAc/hexanes). ${ }^{1} \mathrm{H}$ NMR (400 MHz, $\left.\mathrm{CDCl}_{3}\right) \delta 1.43(\mathrm{~s}, 9 \mathrm{H}), 2.30-2.38(\mathrm{~m}, 2 \mathrm{H}), 2.57-2.75(\mathrm{~m}, 2 \mathrm{H}), 3.87(\mathrm{dd}, J=11.5,3.8 \mathrm{~Hz}$, 1H), 3.95 (dd, $J=11.5,3.8 \mathrm{~Hz}, 1 \mathrm{H}), 4.26-4.34$ (br s, 1H), 4.94-5.06 (m, 2H), 5.57-5.65 (br s, 1H), 5.71-5.84 (m, 1H). $\left.{ }^{13} \mathrm{C} \mathrm{NMR} \mathrm{(100} \mathrm{MHz,} \mathrm{CDCl}_{3}\right) \delta 207.6,155.9,136.7,115.4$, 80.1, 62.6, 61.5, 39.0, 28.2, 27.2. HRMS (EI) m/z $243.1471\left[\mathrm{M}^{+}\right.$; calcd for $\mathrm{C}_{12} \mathrm{H}_{21} \mathrm{NO}_{4}$ : $243.1471] .[\alpha]_{\mathrm{D}}^{20}=+10^{\circ}\left(\mathrm{c} 0.01, \mathrm{CHCl}_{3}\right)$.

\section{tert-Butyl-(1S)-1-[(1R)-hydroxyethyl]-2-oxo-hex-5-enylcarbamate (2k)}

Prepared from methyl ester 1k (489 $\mathrm{mg}, 2.1 \mathrm{mmol})$ and vinyl magnesium bromide $(12.6 \mathrm{mmol})$ in the presence of $\mathrm{Cu}(\mathrm{OAc})_{2}(0.63$

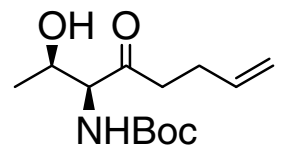

mmol) according to procedure $\mathrm{A}$ (reaction time $12 \mathrm{~h}$ at room temperature) to yield crude product $(512 \mathrm{mg}$ ). Purification by chromatography over basic alumina (activity grade I; 40:60 EtOAc/hexanes increasing to 90:10 EtOAc/hexanes in 10\% increments) gave $2 \mathbf{k}$ as pale yellow oil (265 mg, 49\%). TLC $\mathrm{R}_{\mathrm{f}} 0.46$ (40:60 EtOAc/hexanes). ${ }^{1} \mathrm{H}$ NMR (400 $\left.\mathrm{MHz}, \mathrm{CDCl}_{3}\right) \delta 1.15(\mathrm{~d}, J=6.3 \mathrm{~Hz}, 3 \mathrm{H}), 1.40(\mathrm{~s}, 9 \mathrm{H}), 2.25-2.34(\mathrm{~m}, 2 \mathrm{H}), 2.56-2.78(\mathrm{~m}$, 2H), 4.16 (br d, $J=8.2 \mathrm{~Hz}, 1 \mathrm{H}), 4.22-4.33(\mathrm{~m}, 1 \mathrm{H}), 4.88-5.04$ (m, 2H), 5.45-5.54 (m, $1 \mathrm{H}), 5.68-5.82(\mathrm{~m}, 1 \mathrm{H}) .{ }^{13} \mathrm{C} \mathrm{NMR}\left(100 \mathrm{MHz}, \mathrm{CDCl}_{3}\right) \delta 208.8,156.2,136.8,115.4,80.0$, 
Supporting Information: Org.Lett, One-pot synthesis of homoallylic ketones. Hansford et al.

67.0, 63.9, 39.5, 28.2, 27.2, 19.4. HRMS (EI) m/z 257.1626 [M+' calcd for $\mathrm{C}_{13} \mathrm{H}_{23} \mathrm{NO}_{4}$ : 257.1627]. $[\alpha]_{\mathrm{D}}{ }^{20}=-19^{\circ}\left(\mathrm{c} 0.01, \mathrm{CHCl}_{3}\right)$.

\section{tert-Butyl-(2R,4S)-4-Hydroxy-2-pent-4-enoyl-pyrrolidine-1-carbamate (2l)}

Prepared from methyl ester $11(\mathrm{PG}=\mathrm{Boc})(270 \mathrm{mg}, 1.1 \mathrm{mmol})$ and vinyl magnesium bromide $(4.4 \mathrm{mmol})$ according to procedure $\mathrm{D}$ (reaction time $24 \mathrm{~h}$ at room temperature) to yield crude product (347

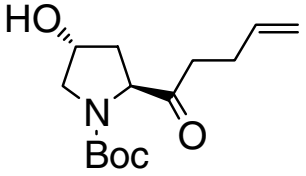
mg). Purification by chromatography over florisil (20:80 EtOAc) gave $2 \mathbf{l}(\mathrm{PG}=\mathrm{Boc})$ as a viscous oil which slowly crystallized on standing, (207 mg, $70 \%)$. Recrystallisation from $\mathrm{Et}_{2} \mathrm{O} /$ hexanes gave colorless needles, mp $104-106{ }^{\circ} \mathrm{C}$. $\quad$ TLC $\mathrm{R}_{\mathrm{f}}$ 0.21 (40:60 EtOAc/hexanes). ${ }^{1} \mathrm{H}$ NMR (400 MHz, $\left.\mathrm{CDCl}_{3}\right) \delta 1.42$ (s, 9H), 1.74-1.83 (br m, 1H), 1.832.02 (br m, 1H), 2.39-2.59 (br m, 1H), 3.13-3.26 (m, 1H), 3.52-3.71 (m, 1H), 4.11 (t, $J=$ 8.2 Hz, 1H), 4.19-4.35 (br m, 1H), 5.08-5.25 (m, 2H), 5.29-5.52 (m, 2H), 5.78-5.99 (m, 2H), 6.49-6.72 (br s, 1H). ${ }^{13} \mathrm{C}$ NMR (100 MHz, $\left.\mathrm{CDCl}_{3}\right) \delta$ 157.9, 139.4, 136.8, 116.2, 115.3, 81.0, 78.6, 68.9, 65.1, 56.3, 37.6, 28.2. HRMS (FAB) m/z 270.1719 [M+H' ; calcd for $\left.\left[\mathrm{C}_{14} \mathrm{H}_{24} \mathrm{NO}_{4}\right]^{+}: 270.1700\right] .[\alpha]_{\mathrm{D}}{ }^{20}=-60^{\circ}\left(\mathrm{c} 0.01, \mathrm{CHCl}_{3}\right)$.

\section{1-[(2R,4S)-4-Hydroxy-1-(9-phenyl-9H-fluoren-9-yl)-pyrrolidin-2-yl]-pent-4-en-1-one $(2 \mathrm{~m})$}

Prepared from methyl ester $\mathbf{1 m}(5.0 \mathrm{~g}, 13 \mathrm{mmol})$ and vinyl $\mathrm{HO}$, magnesium bromide $(65 \mathrm{mmol})$ according to procedure $\mathrm{E}$ (total reaction time ca. 7 days at room temperature) to yield crude product

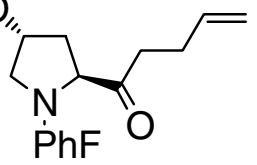
(5.26 g). Purification by chromatography over florisil (20:80 EtOAc/hexanes) gave $\mathbf{2 m}$ as a semi-solid product $(3.74 \mathrm{~g}, 70 \%)$. Trituration of the product with $\mathrm{Et}_{2} \mathrm{O} /$ hexanes $(1: 1)$ 
Supporting Information: Org.Lett, One-pot synthesis of homoallylic ketones. Hansford et al.

produced a white solid which could be collected by filtration. Recrystallization from $\mathrm{Et}_{2} \mathrm{O}$ gave colorless needles, mp 101-103 ${ }^{\circ} \mathrm{C}$. TLC $\mathrm{R}_{\mathrm{f}} 0.35$ (50:50 EtOAc/hexanes). ${ }^{1} \mathrm{H}$ NMR $\left(400 \mathrm{MHz}, \mathrm{CDCl}_{3}\right) \delta 1.35-1.44(\mathrm{~m}, 1 \mathrm{H}), 1.50(\mathrm{~d}, J=6.0 \mathrm{~Hz}, 1 \mathrm{H}), 1.61-1.75(\mathrm{~m}, 2 \mathrm{H})$, 1.85-2.06 (m, 2H), 2.11-2.21 (m, 1H), 2.99 (dd, $J=10.0,5.6 \mathrm{~Hz}, 1 \mathrm{H}), 3.23(\mathrm{dd}, J=9.1$, $5.6 \mathrm{~Hz}, 1 \mathrm{H}), 3.61(\mathrm{dd}, J=10.0,5.4 \mathrm{~Hz}, 1 \mathrm{H}), 4.45$ (sextet, $J=5.9 \mathrm{~Hz}, 1 \mathrm{H}), 4.89$ (t, $J=1.4$ $\mathrm{Hz}, 1 \mathrm{H}), 4.90-4.94(\mathrm{~m}, 1 \mathrm{H}), 5.58-5.69(\mathrm{~m}, 1 \mathrm{H}), 7.05-7.81(\mathrm{~m}, 13 \mathrm{H}) .{ }^{13} \mathrm{C} \mathrm{NMR}(100 \mathrm{MHz}$, $\left.\mathrm{CDCl}_{3}\right) \delta 212.6,148.4,145.5,142.4,141.8,139.4,137.4,128.8,128.4,128.3,127.7$, 127.6, 127.4, 127.3, 126.8, 120.0, 119.7, 114.8, 77.2, 76.4, 70.2, 64.3, 57.1, 38.9, 38.7, 27.1. HRMS $(\mathrm{FAB}) \mathrm{m} / \mathrm{z} 410.2139\left[\mathrm{M}+\mathrm{H}^{+}\right.$; calcd for $\left.\left[\mathrm{C}_{28} \mathrm{H}_{28} \mathrm{NO}_{2}\right]^{+}: 410.2109\right] .[\alpha]_{\mathrm{D}}{ }^{20}=$ $+226^{\circ}\left(\mathrm{c} 0.01, \mathrm{CHCl}_{3}\right)$.

\section{1-(1-Methyl-1H-pyrrol-2-yl)-pent-4-en-1-one (2n)}

Prepared from methyl ester 1n (306 mg, $2.2 \mathrm{mmol})$ and vinyl magnesium bromide $(6.6 \mathrm{mmol})$ according to procedure $\mathrm{D}$ (reaction time $12 \mathrm{~h}$ at room temperature) to yield crude product $(354 \mathrm{mg})$.

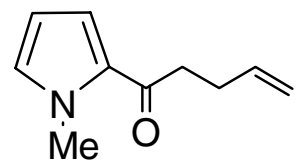
Purification by chromatography over basic alumina (activity grade I; 5:95 Et $2 \mathrm{O} /$ hexanes) gave $2 \mathrm{n}$ as pale yellow oil (128 mg, $36 \%$ ). TLC $\mathrm{R}_{\mathrm{f}} 0.46$ (10:90 Et $\mathrm{E}_{2} \mathrm{O} /$ hexanes). ${ }^{1} \mathrm{H} \mathrm{NMR}$ $\left(400 \mathrm{MHz}, \mathrm{CDCl}_{3}\right) \delta$ 2.37-2.46 (m, 2H), $2.85(\mathrm{t}, J=7.5 \mathrm{~Hz}, 2 \mathrm{H}), 3.91(\mathrm{~s}, 3 \mathrm{H}), 4.93-4.99$ (m, 1H), 5.01-5.09 (m, 1H), 5.79-5.92 (m, 1H), 6.09 (dd, J = 4.0, $2.7 \mathrm{~Hz}, 1 \mathrm{H}), 6.75-6.79$ $(\mathrm{m}, 1 \mathrm{H}), 6.93(\mathrm{dd}, J=4.0,1.7 \mathrm{~Hz}, 1 \mathrm{H}) .{ }^{13} \mathrm{C} \mathrm{NMR}\left(100 \mathrm{MHz}, \mathrm{CDCl}_{3}\right) \delta$ 190.2, 137.5, 130.8, 130.5, 118.8, 114.8, 107.7, 37.9, 37.5, 28.8. HRMS (EI) m/z 163.1007 [M+' calcd for $\mathrm{C}_{10} \mathrm{H}_{13} \mathrm{NO}$ : 163.0997$]$.

\section{Oct-7-en-4-one (2o)}

Prepared from methyl ester $10(1.02 \mathrm{~g}, 10 \mathrm{mmol})$ and vinyl magnesium bromide $(40 \mathrm{mmol})$ in the presence of $\mathrm{Cu}(\mathrm{OAc})_{2}(3 \mathrm{mmol})$ according to

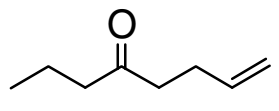
procedure $\mathrm{A}$ (reaction time $12 \mathrm{~h}$ at room temperature) to yield crude product (1.22 $\mathrm{g})$. Purification by chromatography over florisil (hexane) gave $\mathbf{2 0}$ as a pale yellow oil (353 
Supporting Information: Org.Lett, One-pot synthesis of homoallylic ketones. Hansford et al.

mg, $29 \%) .{ }^{1} \mathrm{H}$ NMR (400 MHz, $\left.\mathrm{CDCl}_{3}\right) \delta 0.84(\mathrm{t}, J=8.0 \mathrm{~Hz}, 3 \mathrm{H}), 1.48-1.61(\mathrm{~m}, 2 \mathrm{H})$, $2.25(\mathrm{q}, J=8.0 \mathrm{~Hz}, 2 \mathrm{H}), 2.32(\mathrm{t}, J=8.0 \mathrm{~Hz}, 2 \mathrm{H}), 2.44(\mathrm{t}, J=8.0 \mathrm{~Hz}, 2 \mathrm{H}), 4.87-5.02(\mathrm{~m}$, 2H), 5.66-5.79 (m, 1H). ${ }^{13} \mathrm{C} \mathrm{NMR}\left(100 \mathrm{MHz}, \mathrm{CDCl}_{3}\right) \delta 210.1,137.0,114.9,44.6,41.6$, 27.6, 17.1, 13.6.

\section{tert-Butyl-(2R,3S)-3-hydroxy-hept-6-enyl-carbamate (4)}

A solution of $\mathbf{2 h}(200 \mathrm{mg}, 0.88 \mathrm{mmol})$ in EtOH $(2 \mathrm{~mL})$ was slowly added to a suspension of lithium tri-tert-butoxyaluminohydride (895 $\mathrm{mg}, 3.5 \mathrm{mmol})$ in $\mathrm{EtOH}(15 \mathrm{~mL})$ at $-78^{\circ} \mathrm{C}$. After $2 \mathrm{~h}$, the reaction<smiles>C=CCCC(O)C(C)NC(C)(C)C</smiles>
was poured onto a mixture of citric acid solution (10\% aqueous, $100 \mathrm{ml}) /$ ice (100 g)/EtOAc (50ml) with vigorous stirring. The layers were separated and the aqueous layer was extracted twice with EtOAc/ $\mathrm{CH}_{2} \mathrm{Cl}_{2}$ (3:1). The combined extracts were washed with $\mathrm{HCl}$ solution (1 $M$ aqueous) (x1), $\mathrm{NaHCO}_{3}$ solution (saturated, aqueous) (x1), brine, dried and evaporated to afford crude alcohol 4 (169 mg). TLC $R_{\mathrm{f}} 0.32$ (25:75 EtOAc/hexanes). The ${ }^{1} \mathrm{H}$ NMR spectrum of the crude product (400 MHz) revealed a pair of well resolved methyl doublets (97:3 ratio): $(2 R, 3 S)$-diastereomer (major): $\delta 1.05, J=6.8 \mathrm{~Hz} ;(2 R, 3 R)$ diastereomer (minor): $\delta 1.13, J=6.8 \mathrm{~Hz}$. Recrystallization of the crude product (hexane) gave a diastereomerically enriched sample (d.r. $\geq 99: 1)$, mp 90-91 ${ }^{\circ} \mathrm{C}$.

In an alternative procedure, reduction of compound $\mathbf{2 h}$ with $\mathrm{NaBH}_{4}$ in $\mathrm{MeOH}$ at $0{ }^{\circ} \mathrm{C}$ gave alcohol $\mathbf{4}$ with d.r. $(2 R, 3 S)-\mathbf{4}:(2 R, 3 R)-\mathbf{4}=75: 25$.

Peaks for major diastereomer (2R,3S)-4: ${ }^{1} \mathrm{H}$ NMR (400 MHz, $\left.\mathrm{CDCl}_{3}\right) \delta 1.05(\mathrm{~d}, J=6.8$ $\mathrm{Hz}, 3 \mathrm{H}), 1.40(\mathrm{~s}, 9 \mathrm{H})$ partially overlapped with 1.41-1.49 (m, 2H), 2.02-2.14 (m, 1H), 2.18-2.29 (m, 1H), 2.52 (br s, 1H), 3.58-3.71 (m, 2H), 4.81 (d, J = 7.8 Hz, 1H), 4.93 (d, J 
Supporting Information: Org.Lett, One-pot synthesis of homoallylic ketones. Hansford et al.

$=10.1 \mathrm{~Hz}, 1 \mathrm{H}), 5.01(\mathrm{dd}, J=17.1,1.6 \mathrm{~Hz}, 1 \mathrm{H}), 5.73-5.85(\mathrm{~m}, 1 \mathrm{H}) .{ }^{13} \mathrm{C} \mathrm{NMR}(100 \mathrm{MHz}$, $\left.\mathrm{CDCl}_{3}\right) \delta 156.1,138.4,115.2,79.7,74.1,50.9,32.6,30.5,28.6,14.7 . \mathrm{HRMS}(\mathrm{EI}) \mathrm{m} / \mathrm{z}$ $229.1682\left[\mathrm{M}^{+}\right.$; calcd for $\mathrm{C}_{12} \mathrm{H}_{23} \mathrm{NO}_{3}$ : 229.1678]. $[\alpha]_{\mathrm{D}}{ }^{20}=+16^{\circ}\left(\mathrm{c} 0.01, \mathrm{CHCl}_{3}\right)$.

Peaks for minor diastereomer (2R,3R)-4 (abstracted from 75:25 mixture obtained above):

${ }^{13} \mathrm{C}$ NMR $\left(100 \mathrm{MHz}, \mathrm{CDCl}_{3}\right) \delta 156.4,138.5,115.1,79.5,74.5,50.5,33.4,30.1,28.6$, 18.4.

\section{Determination of enantiomeric purity:}

Example: Synthesis of $\quad\left(2^{\prime} S\right)-N-[(2 R, 3 S)-3-h y d r o x y-h e p t-6-e n y l]-1-$ methylpyrrolidine-2-carboxamide (5)

Alcohol 4 (as a 97:3 mixture of diastereomers, $9.9 \mathrm{mg}, 0.043$ mmol) was treated with a solution of TFA in $\mathrm{CH}_{2} \mathrm{Cl}_{2}(1: 1,1 \mathrm{~mL})$ at $0{ }^{\circ} \mathrm{C}$. After $1 \mathrm{~h}$, the volatiles were removed under reduced pressure, and the resultant residue co-evaporated twice ( $1 \mathrm{~mL}$ each)<smiles></smiles>
from $\mathrm{HCl}$ saturated dioxane solution. The crude product was dissolved in $\mathrm{CH}_{2} \mathrm{Cl}_{2}(1 \mathrm{~mL})$ containing DIPEA $(30 \mu \mathrm{L}, 0.17 \mathrm{mmol})$, and then treated with $(2 R S)$ - or $(2 S)-1$ benzenesulfonyl-pyrrolidine-2-carbonyl chloride $(1.16 \mathrm{~mL}$ of a $0.037 \mathrm{M}$ solution in $\mathrm{CH}_{2} \mathrm{Cl}_{2}$, equivalent to $11.8 \mathrm{mg}, 0.043 \mathrm{mmol}$ ). After $3 \mathrm{~h}$, the volatiles were removed under reduced pressure and the resultant residue taken up into EtOAc. The organic phase was washed with $\mathrm{HCl}$ solution ( $1 M$ aqueous), $\mathrm{NaHCO}_{3}$ solution (saturated, aqueous), brine, dried $\left(\mathrm{MgSO}_{4}\right)$ and evaporated to give the title compound (14.1 mg, 90\%).

A portion was chromatographed (60:40 EtOAc/hexanes) for characterization. Peaks for major diastereomer $(2 R, 3 S, 2 ' S)-5:{ }^{1} \mathrm{H} \mathrm{NMR}\left(400 \mathrm{MHz}, \mathrm{CDCl}_{3}\right) \delta 1.15(\mathrm{~d}, J=6.9 \mathrm{~Hz}$, $3 \mathrm{H}), 1.38-1.82(\mathrm{~m}, 5 \mathrm{H}), 2.04-2.34(\mathrm{~m}, 3 \mathrm{H}), 3.13-3.23(\mathrm{~m}, 1 \mathrm{H}), 3.52-3.60(\mathrm{~m}, 1 \mathrm{H}), 3.61$ $3.67(\mathrm{~m}, 1 \mathrm{H}), 3.95-4.06(\mathrm{~m}, 2 \mathrm{H}), 4.93-4.99(\mathrm{~m}, 1 \mathrm{H}), 5.00-5.08(\mathrm{~m}, 1 \mathrm{H}), 5.75-5.88(\mathrm{~m}$, $1 \mathrm{H}), 6.96(\mathrm{~d}, J=7.7 \mathrm{~Hz}, 1 \mathrm{H}), 7.51-7.58(\mathrm{~m}, 2 \mathrm{H}), 7.61-7.67(\mathrm{~m}, 1 \mathrm{H}), 7.80-7.86(\mathrm{~m}, 2 \mathrm{H})$. 
Supporting Information: Org.Lett, One-pot synthesis of homoallylic ketones. Hansford et al.

Analagous signals for $\left(2 R, 3 S, 2^{\prime} R\right)-\mathbf{5}$, if present in the crude product, were partially obscured by a small impurity peak which appeared in the vicinity of the methyl doublet for $\left(2 R, 3 S, 2^{\prime} R\right)-5$, as shown by chemical shift comparison. Thus, diastereomeric alcohol mixture $(2 R, 3 S)-\mathbf{4}:(2 R, 3 R)-\mathbf{4}=75: 25$ (obtained from $\mathrm{NaBH}_{4}$ reduction of $\mathbf{2 h}$ as described above) was deprotected and coupled to $(2 R S)$-1-benzenesulfonyl-pyrrolidine-2-carbonyl chloride in a manner analogous to that described above, to give a 3:3:1:1 ratio of $\left(2 R, 3 S, 2^{\prime} R\right)-5:\left(2 R, 3 S, 2^{\prime} S\right)-5:\left(2 R, 3 R, 2^{\prime} S\right)-5:\left(2 R, 3 R, 2^{\prime} R\right)-5$. Each diastereomer gave a well resolved methyl doublet in the ${ }^{1} \mathrm{H}$ NMR spectrum $\left(\mathrm{CDCl}_{3}, 400 \mathrm{MHz}\right)$ at $\delta 1.13,1.15$, 1.19 and 1.22 .

To confirm that the impurity peak did not arise from diastereomer $\left(2 R, 3 S, 2^{\prime} R\right)-5$, the 3:3:1:1 diastereomeric mixture $\left(2 R, 3 S, 2^{\prime} R\right)-\mathbf{5}:\left(2 R, 3 S, 2^{\prime} S\right)-\mathbf{5}:\left(2 R, 3 R, 2^{\prime} S\right)-\mathbf{5}:\left(2 R, 3 R, 2^{\prime} R\right)-5$ was incrementally added to $(2 R, 3 S, 2 ' S)-5$. At levels approaching $1-2 \%$, diastereomeric signals for $\left(2 R, 3 S, 2^{\prime} R\right)-5$ were clearly visible, as shown by the appearance of the upfield methyl doublet at $\delta 1.13$.

\section{$(4 R, 5 S)-5-B u t-3-e n y l-4-m e t h y l-o x a z o l i d i n-2-o n e ~(6)$}

A solution of alcohol 4 (as 99:1 mixture of diastereomers, $30 \mathrm{mg}$, $0.031 \mathrm{mmol})$ in DMF (1 mL) was added to a stirred suspension of $\mathrm{NaH}(60 \%$ dispersion in mineral oil; $27 \mathrm{mg})$ in $\mathrm{DMF}(0.5 \mathrm{~mL})$ at 0 ${ }^{\circ} \mathrm{C}$, and the resultant mixture was stirred for $3 \mathrm{~h}$ at room temperature.

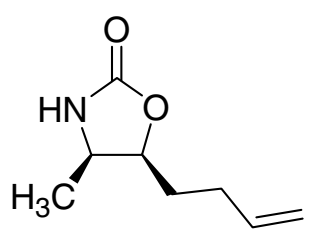
The reaction mixture was cooled to $0{ }^{\circ} \mathrm{C}$, treated with $\mathrm{NH}_{4} \mathrm{Cl}$ solution (saturated aqueous, $2 \mathrm{~mL})$, diluted with water $(5 \mathrm{~mL})$, and extracted with a mixture of $\mathrm{Et}_{2} \mathrm{O} / \mathrm{CH}_{2} \mathrm{Cl}_{2}$ (2:1). The organic phase was washed with water ( $\mathrm{x} 2$ ), brine (x 1), dried $\left(\mathrm{MgSO}_{4}\right)$ and evaporated to give crude product $(30 \mathrm{mg})$. Purification by chromatography over silica (50:50 EtOAc/hexanes) gave pure title compound $(14.4 \mathrm{mg}, 71 \%)$ as a colorless solid. TLC $R_{\mathrm{f}} 0.35$ (60:40 EtOAc/hexanes). ${ }^{1} \mathrm{H}$ NMR (400 MHz, $\left.\mathrm{CDCl}_{3}\right) \delta 1.13(\mathrm{~d}, J=6.5 \mathrm{~Hz}$, $3 \mathrm{H}), 1.52-1.62(\mathrm{~m}, 1 \mathrm{H}), 1.77-1.88(\mathrm{~m}, 1 \mathrm{H}), 2.07-2.19(\mathrm{~m}, 1 \mathrm{H}), 2.23-2.34(\mathrm{~m}, 1 \mathrm{H}), 3.89$ $(\mathrm{q}, J=6.7 \mathrm{~Hz}, 1 \mathrm{H}), 4.52-4.60(\mathrm{~m}, 1 \mathrm{H}), 4.97-5.02(\mathrm{~m}, 1 \mathrm{H}), 5.02-5.09(\mathrm{~m}, 1 \mathrm{H}), 5.72-5.84$ 
Supporting Information: Org.Lett, One-pot synthesis of homoallylic ketones. Hansford et al.

(m, 1H), 5.91 (br s, $1 \mathrm{H}) .{ }^{13} \mathrm{C}$ NMR (100 MHz, $\left.\mathrm{CDCl}_{3}\right) \delta 159.6,136.9,115.8,79.2,51.0$, $29.9,28.4,15.9$.

\section{References}

(1) Vogel, A. I. In Vogel's Textbook of Practical Organic Chemistry, $5^{\text {th }}$ ed.; Furniss, B. S.; Hannaford, A. J.; Smith, P. W. G.; Tatchell, A. R., Rev.; Longman: London, 1989; p 539.

(2) Maurer, P. J.; Takahata, H.; Rapoport, H. J. Amer. Chem. Soc. 1984, 106, 10951098. 